Théologiques

Théologiques

\title{
Le sacré et le territoire slovène
}

\section{Erika Prijatelj}

Volume 16, numéro 1, 2008

Le territoire et le sacré

URI : https://id.erudit.org/iderudit/019184ar

DOI : https://doi.org/10.7202/019184ar

Aller au sommaire du numéro

\section{Éditeur(s)}

Faculté de théologie et de sciences des religions, Université de Montréal

\section{ISSN}

1188-7109 (imprimé)

1492-1413 (numérique)

Découvrir la revue

\section{Citer cet article}

Prijatelj, E. (2008). Le sacré et le territoire slovène. Théologiques, 16(1), 39-63. https://doi.org/10.7202/019184ar

\section{Résumé de l'article}

La notion du sacré s'étend de la réalité substantielle à l'illusion fonctionnelle. Selon nous, la personne humaine en tant qu'être transcendant est un être sacré pour qui la religiosité est un phénomène irréductible; cette personne est aussi un être social. La culture concrétise cette double réalité dans un État, dans une nation. Après 1945, en plus de voir leur existence nationale menacée par une Yougoslavie impérialiste, les Slovènes subirent un régime communiste totalitaire qui voulait créer une société sans classes et un homme libre, mais cette société est plutôt devenue un " sacré " nourri de collectivisme et d'individualisme. L'État et la nation slovènes, en refusant un droit de dénominateur commun à la religion traditionnelle et au mythe de la Narodno Osvobodilna Borna (Lutte pour la libération nationale), se sont soumis aux effets du postmodernisme en raison de leur propre incapacité à vivre leur identité. En se livrant au polycentrisme des valeurs et au plus petit dénominateur commun de la convivialité, les citoyens se reconnaissent aujourd'hui davantage dans la mondialisation que dans les idéaux de leur propre nation. 


\title{
Le sacré et le territoire slovène
}

\author{
Erika PRIJATELJ* \\ Faculté de théologie \\ Université de Ljubljana
}

Tout au long de l'histoire de la nation slovène, le sacré, selon la conception de Durkheim, s'est développé, de la façon propre à un petit agrégat, sur un territoire dont la dimension géographique a considérablement varié. L'identité slovène a ainsi évolué sous l'influence extérieure de nombreuses cultures environnantes et sous l'influence intérieure de la dynamique de sa propre communauté; elle a glissé, particulièrement depuis l'expérience communiste, d'un fondement chrétien à une conception plus sécularisée.

Nous ferons d'abord un bref historique du territoire slovène. Puis nous présenterons quelques préliminaires théoriques pour la compréhension du sacré, puis du sacré en lien avec la personne et la nation, selon une approche essentialiste, tout en cherchant à y intégrer la manière dont la réalité sociale et les phénomènes sociaux se sont "construits ". Dans les deux parties suivantes, selon la même approche, nous situerons l'Europe et la Slovénie après 1945 , puis le sacré et la nationalité slovène dans ce contexte récent. Finalement, nous nous interrogerons pour savoir si la nouvelle systématisation globale des valeurs de la nation et son polycentrisme sont devenus une nouvelle porte d'entrée pour la chrétienté post-traditionnelle et pour la régénération de son contenu.

\section{Repères historiques concernant le territoire slovène}

État d'Europe centrale, limité au nord par l'Autriche, à l'ouest par l'Italie, au sud par la Croatie, à l'est par la Croatie et la Hongrie, la Slovénie est

* $\quad$ Erika Prijatelj est professeur assistant à la Faculté de théologie de l’Université de Ljubljana dans le domaine pastoral et kérygmatique. Ses recherches portent principalement sur le développement de la foi au cours des différentes phases de la vie et dans différentes cultures. Elle a publié plusieurs livres et articles scientifiques en Europe, dans des pays de langue slave ou germanique. 
devenue indépendante en 1991 et a présidé, en première demie de l'année 2008, l'assemblée des vingt-sept États de l'Union européenne. Le pays couvre une superficie de $20255 \mathrm{~km}^{2}$ et compte une population de deux millions d'habitants.

La première formation nationale slovène connue est la Carantanie, célèbre pour son organisation démocratique, son système judiciaire, l'élection populaire de ses princes et les droits progressistes des femmes. Elle a pris place entre 630 et 745 , avec comme centre le château Krn près de Klagenfurt en Autriche. Le territoire national slovène a connu sa plus grande superficie au XIX siècle, soit $70000 \mathrm{~km}^{2}$.

Entre le XII ${ }^{e}$ siècle et le début du XXe (1918), le destin des terres slovènes est lié à celui de la dynastie des Habsbourg. Ces terres constituent une part des possessions héréditaires de la lignée à partir du XIV eiècle. Ainsi, les pays peuplés de Slovènes ne sont plus morcelés ou dispersés, mais, en contrepartie, l'affirmation du pouvoir central à Vienne entraîne l'affaiblissement de l'autonomie des provinces slovènes. Dans le même temps, la germanisation des terres est amorcée: l'allemand devient la langue des élites, tandis que le slovène est progressivement relégué au rang de dialecte parlé par la paysannerie. La germanisation, par des annexions territoriales successives en faveur de l'Autriche, fut aussi une des causes du rétrécissement du territoire.

Au Moyen Âge, du fait de la proximité de l'Empire ottoman, les provinces slovènes subirent pendant de longues années la pression des Turcs, qui, des Balkans, envahissaient les contrées européennes.

En 1848, un groupe d'intellectuels mit au point un programme politique nommé Slovénie unie, qui réclamait l'union des provinces slovènes ainsi que l'instauration du slovène dans les écoles et les offices gouvernementaux. Ce groupe fut marqué de l'influence de divers courants - notamment le panslavisme - et se rapprocha progressivement d'un projet d'union avec les peuples slaves du Sud.

Le développement de la langue slovène sur ce territoire ne s'est toutefois pas fait en opposition aux autres langues slaves du reste des Balkans, mais par rapport aux langues germaniques - on pourrait même dire contre elles. Le premier grand diffuseur du slovène fut le luthérien Primož Trubar, qui traduisit la Bible. De même, le reste des Balkans eut très peu à voir avec l'œuvre du poète France Prešeren qui, au XIX ${ }^{\mathrm{e}}$ siècle, fit prendre conscience aux Slovènes que leur langue pouvait être reconnue dans l'Europe entière grâce à des travaux littéraires de qualité. 
Au milieu du XIX ${ }^{\mathrm{e}}$ siècle, se produisit un bouillonnement confus, alimenté par une idée aussi bien slovène que croate et serbe; ce bouillonnement fut d'autant plus puissant que les Serbes ajoutaient à l'idée un petit État arraché de haute lutte aux Turcs. Très vite ce petit État se perçut comme l'État «naturel» de tous les Slaves du Sud, comme si l'histoire des Serbes, des Croates et des Slovènes, séparés depuis des siècles, ne signifiait plus rien. Le malentendu « yougoslave » commençait à Belgrade, à Zagreb ainsi qu'à Ljubljana.

En 1918, la Slovénie devint une partie du nouveau royaume yougoslave. Les règlements territoriaux consécutifs à la Première Guerre mondiale furent très défavorables à la Slovénie, qui ne compta plus désormais que $30 \%$ de son territoire du IX ${ }^{\mathrm{e}}$ siècle, puisqu'elle dut céder Klagenfurt à l'Autriche, ainsi que l'Istrie et les Alpes juliennes à l'Italie (régions peuplées de 1.300.000 Slovènes).

À partir de 1941, la Slovénie fut divisée en trois régions : le Sud, jusqu'à la capitale Ljubljana, fut annexé par l'Italie, le Nord, de même qu'une petite partie au nord-est, furent occupés par l'Allemagne et la Hongrie (Kos 1996, 9-220; Vodopivec 2007, 9-18).

En 1945, s'ouvrit la période du socialisme d'obédience marxiste. Cette période fait l'objet principal de notre propos.

\section{Quelques préliminaires pour la compréhension du sacré}

La notion du sacré recouvre toutes les options depuis la réalité substantielle jusqu'à l'illusion fonctionnelle. On pourrait l'examiner aussi bien dans un contexte national qu'individuel. Selon notre approche, la notion du sacré perçoit le religieux, à la manière d'Otto, comme un phénomène irréductible ${ }^{1}$. Le sacré signifie surtout ce qui rachète dans le contexte de la religion du salut, ce qui fait appel au dépassement de l'accidentel et du profane, ce qui donne de la signification à l'existence, ce qui lui ajoute de la valeur, ce qui la justifie et la délivre du non-sens et du vide, puisque, selon Heidegger, on ne peut être sauvé que par un dieu. La phénoménologie du sacré montre aussi que celui-ci est une des dimensions essentielles pour une

1. Selon Otto, la religion, ou plutôt la foi en Dieu, n'est pas qu'une sous-division de la connaissance humaine. De même, elle n'est pas qu'un cas particulier de morale ou de philosophie pratique qui poserait une hypothèse sur Dieu en vue d'asseoir ses principes sur une plus grande autorité. La religion englobe des éléments reliés autant à la connaissance qu'à la morale, mais elle ne se réduit à aucune des deux (Otto 1993, 50). 
juste compréhension de la religion et de Dieu². Pour Tillich, un discours sur Dieu qui n'inclut pas la catégorie du sacré est non seulement du non-sacré mais aussi du non-vrai. Sans la catégorie du sacré, Dieu devient un objet séculier et un naturaliste aurait alors raison de nier l'existence d'un tel Dieu. Tout discours sur le sacré qui n'est pas connecté à un discours sur le divin transforme le sacré en sentiment religieux ascétique (Hollis 1995, $147)^{3}$. Le sacré est l'apanage de ce qui est l'ultime fondement de l'homme, car seul le sacré peut lui donner l'ultime réponse et seule l'ultime réponse de l'homme dans sa situation existentielle peut inclure la catégorie du sacré. C'est précisément à cause de sa transcendance et de son caractère paradoxal qu'il dépasse la structure sujet-objet et qu'ainsi il échappe à la compétence rationnelle du sujet (Tillich 1951, 217).

Le sacré, dans sa signification authentique en regard du divin comporte une certaine ambiguïté par rapport à la transcendance. Il n'est pas ou, du moins pas tout à fait, de l'ordre du tout autre ni de l'ordre de la transcendance au sens absolu du terme, et il ne s'aventure pas au-delà de cette existence de façon radicalement différente. Néanmoins, il suppose un retrait

2. Contrairement à des auteurs slovènes plus connus comme Stres ou Jamnik, un autre philosophe slovène, Hribar, influencé par la critique marxiste de la religion, reproche à Heidegger une erreur essentielle : celle d'avoir fait dériver le divin du sacré. Selon Hribar, Heidegger a fait la rencontre du sacré et il lui a donné ce sens bien défini. Toutefois, dans la phase finale de sa réflexion, Heidegger s'est éloigné de l'essentiel du sacré qu'il traita dorénavant en lien avec le divin, et c'est ainsi qu'il laissa tomber le monde comme l'espace de l'entité (voir Hribar 1986).

3. Otto parle de sentiment religieux comme d'un a priori objectif de chacun des phénomènes religieux qui a son objet hors de l'homme; il s'agit d'un objet numineux et c'est ainsi qu'il maintient ce qui est essentiel pour la religion: son orientation vers la transcendance, vers le tout autre. Lorsque Otto parle de ce sentiment, il parle d'une expérience affective du sacré, expressément orientée au-delà du sujet et qui a un contenu spécifique que nous nommons numineux, et qui est en même temps mysterium tremendum et fascinans. Le sens du mot numineux met l'accent sur le sui generis du sacré, c.-à-d. sur son caractère non rationnel et non moral, car Dieu, comme réalité transcendante, ne peut pas être un objet parmi d'autres objets, et c'est pour cela que la transcendance est en lien avec le mystère. La moralité et la rationalité font partie du sacré dans les religions développées et théistes (voir The New Encyclopaedia Britannica 1985, 560). 
du domaine du profane ${ }^{4}$ et de tout ce que l'homme arrive à maîtriser par la technique. Il diffère de l'existence d'ici-bas, il suppose même l'existence d'un au-delà, ce que l'entité lui permet. En ce sens, il est tout juste un peu moins qu'un dieu religieux. Il présume une profondeur non-définie de l'existence, mais l'idée de Dieu contient aussi de l'au-delà, du tout autre sublime, et elle est plus complexe que celle du sacré, car elle englobe de façon comparable les deux dimensions symboliques du divin et du sacré. Le sacré et l'idée de Dieu ne se recoupent que partiellement en raison précisément de la symbolique de la majesté sublime de Dieu. On ne peut donc pas placer dans un rapport d'équivalence Dieu et le sacré (Vergote 1952, 151; Tamarut 2005, 43-45).

En plus de son sens religieux et philosophique, l'idée du sacré joue un rôle dans la vie quotidienne de la personne et de la nation, là où s'insèrent deux sortes de phénomènes: ceux qui ont un lien évident avec le surnaturel religieux et qui sont pour cela dépendants de la façon dont la religion les traite, et ceux qui ne sont pas reliés au religieux mais que pourtant nous nommons sacrés, comme, par exemple, l'amour de sa patrie, le respect de sa langue maternelle, la vénération de ses parents, le culte des morts, etc. La réalité socialement construite est alors vue comme un processus dynamique.

\section{Le sacré, la personne et la nation}

Pour saisir ce qui s'est passé sur le territoire slovène au $\mathrm{XX}^{\mathrm{e}}$ siècle, nous voulons d'abord présenter notre conception de la personne. Elle est, per se, un être sacré, parce que transcendant : telle est notre prémisse. Même si, dans un sens plus restreint, nous utilisons la notion de transcendance pour l'idée religieuse de Dieu, celle-ci s'applique aussi à l'homme. L'aspect

4. En fait, le sacré ne se définit que par son opposition avec le profane. Cette opposition est, en effet, d'un genre tout particulier: elle est absolue et telle qu'aucune autre ne peut lui être comparée. Cette hétérogénéité radicale se traduit par des signes spéciaux: parce que nous sommes habitués à concevoir dans notre esprit un vide logique entre le sacré et le profane, nous éprouvons une insurmontable répugnance à penser qu'ils puissent se trouver en contact d'une manière quelconque; et de là résulte toute la série des interdictions de contact direct entre eux, ces interdictions s'étendant plus ou moins loin selon l'intensité du caractère sacré des choses que l'on considère: interdiction du contact matériel, du contact par le regard, ou par la parole (silence religieux); interdiction de les mêler dans le temps, ou même de les rapprocher par la pensée (Durkheim 1907, 9). 
anthropologique le plus typique de la dignité humaine ${ }^{5}$ et de sa valeur absolue est la transcendance, de sorte qu'il paraît inadmissible que la personne puisse être soumise au service de l'autre ou devenir un objet jetable après usage. Considérant que la personne est son propre but, elle dépasse son inclusion dans l'environnement, dans le temps et dans l'espace et, d'une certaine manière, elle se dépasse elle-même. "Les citoyens sont des êtres qui partagent des territoires dont la physique, en tant que science natu-

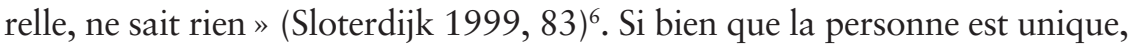
irremplaçable; elle possède une histoire personnelle qui, au fil des ans, s'est développée sur un territoire bien déterminé. Mais, en vertu même de sa transcendance, l'homme est libre et responsable de lui-même. Ainsi se dessine un sens de la liberté qui implique que ce dernier peut, dans son environnement, agir pour maîtriser sa destinée, sans pour autant se contenter de ne satisfaire que ses fantaisies. Le sens de sa liberté se manifeste alors dans le fait que l'homme n'est plus seulement le produit de forces extérieures; il devient un être autonome qui évolue dans la dignité. C'est seulement de cette façon qu'il devient un être culturellement apte à créer du neuf.

Les trois expressions, la dignité de la personne, la personne comme valeur absolue et la personne comme but et non pas comme moyen, se recoupent dans ce qu'elles interdisent: la manipulation et l'utilisation de l'autre. En conséquence, les droits humains qui se portent garants de la

5. La théorie de Kant sur la dignité de la personne s'appuie sur l'autonomie de l'homme. Il dit explicitement que la dignité de l'être raisonnable réside dans le fait qu'il n'est pas soumis à d'autre loi qu'à celle qu'il se donne (Honnenfelder 1996, 239). Sa dignité est alors interprétée de manière individualiste ou même idéaliste parce qu'elle s'appuie sur l'idée de la propre justification de soi-même. Le $\mathrm{xx}^{\mathrm{e}}$ siècle a essayé de dépasser ce point de vue. La phénoménologie et la philosophie du dialogue mettaient l'accent sur l'ouverture fondamentale et constitutive de la personne; Heidegger et Lévinas découvrent l'homme comme un être radicalement dépossédé et décentralisé. L'idée fondamentale de la dignité de la personne n'est donc plus dans la justification de l'homme par lui-même, mais dans sa transcendance qui vient de son ouverture vers les autres et vers l'Autre.

6. L'œuvre de Sloterdijk met l'accent sur le sens du territoire et de l'habitat de la personne. D'abord, la personne commence à vivre dans un placenta et cette première détermination accompagne l'homme tout au long de sa vie. Il se détache/s'attache continuellement, et il a besoin en permanence d'un cadre et d'un espace vital qui sont pour lui une source de stimulation et d'énergie, comme le sont la nourriture et l'information. En raison de sa connexion avec le territoire, la personne est un être « tendu entre la particularité, la totalité ou la généralité; ce qui se présente aujourd'hui comme un problème d'individualisme, de collectivisme ou de holisme» (Hollis 1995, 147). 
liberté de conscience et de la liberté religieuse ou idéologique sont inaliénables. Une nation et sa culture justifient leur raison d'être quand elles permettent à la personne de devenir, à son gré et y compris à travers sa relation avec le sacré, un être exclusif, libre et autonome; car c'est sa propre culture, dont la personne ne peut jamais faire abstraction, qui la qualifie et la construit ${ }^{7}$.

En même temps que le caractère sacré de sa dignité différencie la personne, cette dernière est aussi, par définition, un être social; et la culture concrétise cette réalité dans un État, dans une nation, dans une religion, dans des coutumes, dans des normes, dans des associations, dans un territoire, etc. Dans ce contexte, le religieux ne serait alors rien d'autre qu'un tissu de relations que l'homme construit avec le sacré. La socialité exprime une condition nécessaire à la culture, car c'est de cette dernière que l'homme reçoit son identité et devient homme. «Il ne peut justifier son individualité qu'à l'intérieur du cadre culturel qui s'avère en même temps la condition nécessaire pour s'affirmer lui-même dans sa dignité » (Juhant et Žalec 2006, 20). Au début de son étude sur les origines de la vie religieuse, Durkheim définit, mais non exclusivement, la religion comme "un système de croyances et de pratiques relatives à des choses sacrées - croyances et pratiques communes à une collectivité déterminée. » La religion lui apparaît comme un phénomène social (Durkheim 1907, 4).

En fait, le problème de fond est celui de la juste compréhension des fondements sacrés des religions — du moins en modernité — sur un territoire donné, et de leur place dans une société démocratique, libérale et laïque. Habituellement, les données sociales fondamentales sont la famille, la nation et l'État, à l'intérieur desquels s'accomplit et se transmet l'activité

7. En ce sens, l'allocution prononcée par Jean-Paul II, au siège de l'UNESCO à Paris, en pleine période communiste de l'ancienne Yougoslavie et d'autres pays d'Europe, rejoint nos préoccupations: "La culture est ce par quoi l'homme en tant qu'homme devient davantage homme, "est" davantage, accède davantage à "l'être" ". Plus loin, tout en soulignant que «l'homme ne peut se passer de la culture », le pape mettra en relief le rôle social et national de la culture, laquelle est une force, une puissance: "L'homme vit toujours selon une culture qui lui est propre et qui, à son tour, crée entre les hommes un lien qui leur est propre aussi, en déterminant le caractère interhumain et social de l'existence humaine. Dans l'unité de la culture comme mode propre de l'existence humaine, s'enracine en même temps la pluralité des cultures au sein de laquelle l'homme vit. Pour créer la culture, il faut considérer, jusque dans ses dernières conséquences et intégralement, l'homme comme une valeur particulière et autonome, comme le sujet porteur de la transcendance de la personne » (Jean-Paul II 1980, 602-608). 
humaine. Ces cellules sociales sont les bases sur lesquelles l'homme s'appuie pour se réaliser; mais en même temps elles annoncent les limites de sa liberté: la société favorise la liberté autant qu'elle la tempère. Il revient à l'État et à toutes ses institutions de garantir et de consolider la dimension sociale humaine et, en même temps, de protéger l'homme de sorte que les encadrements sociaux non seulement ne le dépossèdent pas mais, au contraire, l'aident dans le développement de la société. «Le principe du fonctionnement de la démocratie va du bas vers le haut; inversement, l'ordre social totalitaire fonctionne du haut vers le bas, ce qui signifie que cet ordre est abstrait, non personnel, non humain et non pratique, parce qu'il ne prend pas en considération le fait que l'homme peut être son propre pourvoyeur de soin» (Juhant 2006, 264).

De nombreuses communautés ont éprouvé le besoin de fondements sacrés, besoin exprimé par un culte, pour se situer dans l'existence ${ }^{8}$. C'est en les explorant que nous découvrons l'influence durable qu'ils ont exercée sur les identités nationales. À première vue, cette affirmation pourrait, de nos jours, sembler paradoxale. Du moins en Occident, où certaines valeurs reliées plutôt au confort matériel ont érodé le sens du sacré et écarté les croyances anciennes en des valeurs transcendantes. Les identités que nous essayons d'y bâtir sont d'abord pragmatiques. Elles s'appuient sur des données économiques monnayées dans une expression politique visant à l'acquisition accélérée de biens matériels. Notre culture évolue sous l'influence du dieu commerce dont l'objectif premier n'est pas d'entretenir une relation privilégiée avec la dimension transcendante du sacré. L'au-delà de l'immédiateté et de ses formes phénoménales à court terme ne l'intéresse pas davantage. D'un côté, la mondialisation a abattu les frontières nationales et s'est ouverte à de nombreuses options, comme celles, par exemple, du développement de l'emploi et de la répartition du travail à l'échelle mondiale, dans la mesure même où ils élargissent le marché et réorganisent, en

8. «Tel est le fondement solide qui fait la valeur de toutes les religions; les cultes ne sont pas des aberrations; ils ont leurs raisons profondes dans la nature même des choses; pendant les cérémonies religieuses où les membres du groupe sont assemblés, le contenu des consciences change; les représentations individuelles, égoïstes, qui les occupent durant les heures de la vie commune, sont chassées obligatoirement parce qu'elles sont profanes; les croyances collectives les remplacent; les intérêts communs sont alors envisagés (exemple: amener la fécondité de l'espèce), de sorte que, pendant ces périodes, l'existence de la société est plus réelle et plus intense qu'en temps ordinaire. Les hommes ne s'abusent donc pas quand ils pensent qu'à ces moments vit quelque chose de plus fort qu'eux dont ils dépendent, qui les hausse audessus d'eux-mêmes, les ennoblit, les sanctifie» (Durkheim, 1907, 5). 
village global, des territoires, des nations et des continents. De l'autre, le processus actuel de la mondialisation représente, pour de nombreuses cultures nationales et leur économie locale, un défi de plus en plus grand si elles veulent conserver leur spécificité. Cela peut les conduire au nationalisme ${ }^{9}$, mais aussi à la xénophobie, à l'antisémitisme, à la discrimination sur la base de la race, de l'âge, du sexe, de l'appartenance ethnique, de la confession religieuse, de l'incapacité physique ou mentale. Cela peut aussi les mener à des débordements internes plus ou moins violents dont nous sommes les témoins, encore de nos jours, dans plusieurs pays, y compris les Balkans. «Jadis, il existait quatre catégories fondamentales pour parler du sacré de la nation: le territoire, la communauté, l'histoire et la destinée " (Smith 2005, 177). Il nous apparaît qu'elles sont encore recevables aujourd'hui, bien qu'avec quelques nuances. On peut les présenter de la façon suivante: 1) une conviction selon laquelle le peuple est un peuple choisi et qu'une mission particulière lui est confiée ou qu'il a un lien exclusif avec une divinité $^{10} ; 2$ ) un attachement au territoire, à la patrie d'ancêtres promus au rang de héros et pour lesquels on a construit des tombeaux et élevé des monuments ${ }^{11}$; 3) une mémoire collective où sont encore présents un âge d'or de l'ethnohistoire nationale et une certaine splendeur matérielle, spirituelle et artistique; 4) un culte des morts rendu aux personnalités célèbres

9. L'essentiel du nationalisme se définit comme une forme de symbolique politique, de culture de masse politisée qui essaie de conditionner les citoyens à aimer leur nation, à respecter ses lois et à défendre leur patrie. «Ce sont les institutions nationales qui forment le génie, le caractère, les goûts et les mœurs d'un peuple, qui lui inspirent cet ardent amour de la patrie» (Rousseau 1915, 431). On pourrait distinguer la dérive nationaliste du patriotisme de la façon suivante: cette dérive glorifie une relation méprisante envers d'autres nations et cultures alors que le patriotisme est l'amour légitime de sa patrie. Le vrai patriotisme, ce qui est bien pour la nation, n'essaie jamais de faire valoir ce qui serait un préjudice à l'égard d'une autre nation (JeanPaul II 1995, 921).

10. Quelques peuples ont inscrit le mot Dieu dans leur hymne, comme les Anglais : "God Save the Queen » (Que Dieu protège la reine) ou les Serbes: «Bože pravde » (Les affaires de Dieu). D'autres l'ont gravé sur leur monnaie, comme les Américains : "In God We Trust» (Nous mettons notre confiance en Dieu). Des bataillons allemands l'ont dessiné sur leur étendard: "Gott mit Uns» (Dieu est avec nous). Les Croates l'ont inscrit dans leur devise: "Bog i Hrvati » (Dieu et Croates), de même que les Slovènes: «Domovina - Mati - Bog" (Patrie - Mère - Dieu).

11. Les Slovènes les plus connus sont, entre autres, le poète France Prešeren, l'écrivain Ivan Cankar, l'architecte Jože Plečnik, le mathématicien Jurij Vega, la sainte Ema Krška, le bienheureux Anton Martin Slomšek et le vénérable Friderik Baraga, missionnaire auprès des Amérindiens du Michigan. 
dont les hauts faits récents ont contribué à la bonne fortune de la nation ${ }^{12}$. Il s'agit là non seulement de caractères sacrés (Armstrong 1997, 597-606; Smith 2000, 791-814), mais aussi de caractères constitutifs, bien que l'une ou l'autre de ces catégories soit sujette à caution comme ce fut le cas en Allemagne où les premiers nationalistes allemands se sont servi, dans leur démonstration et dans leurs cérémonies, d'éléments et de rituels liturgiques spécifiquement chrétiens. Parfois, l'attachement au territoire, perçu comme sacré, s'est avéré le point central de l'existence d'une nation, là où des conflits territoriaux ont sévi pendant longtemps. Dans les contrées où les habitants ethno-nationaux firent d'emblée appel aux quatre catégories sacrées, une identité nationale particulièrement forte et durable s'est développée et il s'ensuivit que la frontière les séparant du monde extérieur fut très marquée. En revanche, là où une ou plusieurs catégories sacrées régressèrent, l'identité nationale s'estompa. Toutefois, l'accueil des étrangers y fut plus ouvert (Smith 2005, 175-179).

Nous entendons le plus souvent le terme nation dans le sens d'une communauté de personnes possédant une culture commune, habitant un territoire spécifique et partageant une même langue, une même économie. La nation est, dans sa substance même, une importante communauté de personnes, c'est à-dire d'êtres transcendants unis par divers liens, avant tout culturels $^{13}$. La nation s'est ainsi constituée comme une communauté dont l'histoire dépasse celle d'un individu ou d'une famille. L'enseignement social catholique est en désaccord avec la conception libérale de la nation qui soutient que ce qui fait qu'une nation est une nation se limite à parler la même langue ou à appartenir au même groupe ethnique, au risque d'omettre que, par le passé, les gens ont bâti de grandes choses ensemble et qu'ils veulent encore les reproduire dans l'avenir. Cette approche libérale de la nation ne retient

12. Lors de la période communiste de l'ancienne Yougoslavie, dont la Slovénie était l'une des républiques, beaucoup de héros nationaux slovènes furent créés qui avaient tous une référence à la personne et à l'idéologie du maréchal Josip Broz Tito. On peut mentionner les Tone Tomšič, Ivan Maček-Matija, Edvard Kardelj et Tito lui-même, slovène par sa mère.

13. Quant à la communauté des souvenirs et des occupations, c'est elle qui donne naissance aux confréries, aux corporations politiques, économiques ou religieuses, où se trouvent réunis tous ceux qui s'adonnent aux mêmes fonctions, ont les mêmes croyances, ressentent les mêmes besoins, etc. (Durkheim 1889, 4). 
que deux composantes, aussi essentielles soient-elles : la conscience nationale et la volonté d'être ou de devenir une nation; la nationalité devient alors une chose que l'homme peut choisir et vouloir construire à son gré, ou au gré des circonstances (Stres 1991, 279).

De plus, l'enseignement catholique sur la nationalité et sa culture ne partage pas le point de vue nationaliste qui absolutise sa propre nationalité et qui y voit une valeur suprême, de telle sorte qu'il est prêt à lui soumettre tout, même la conscience des individus. Cet enseignement reconnaît que la nationalité et la culture ont une valeur indispensable, mais il s'oppose à tout nationalisme qui tôt ou tard s'égare et foule au pied de façon despotique des nations et des cultures, autant celles des autres que les siennes.

\section{L'Europe et la Slovénie après 1945}

La Seconde Guerre mondiale a eu, entre autres effets, celui de renforcer l'antagonisme idéologique de nombreuses nations européennes. Les vainqueurs décidèrent de reconsidérer la situation créée par le Traité de Versailles et de réaménager les frontières. Pour stabiliser le gouvernement de la société internationale, les États-Unis d'Amérique et l'Union soviétique s'y partagèrent la suprématie sur les pays de leur zone d'influence. Ainsi l'Europe fut-elle divisée en deux blocs radicalement opposés. Le principe de la nationalité liée à un territoire bien circonscrit cessa à nouveau d'être un sujet inviolable de la vie politique européenne. À l'est, l'Union soviétique reconnut aux nationalités une existence de transition provisoire lors de la période qui mit fin à l'épisode de la société bourgeoise, progressivement remplacée à son tour par la société socialiste. Il était inévitable, pensait-on, qu'à brève échéance on verrait les nationalités disparaitre au profit de la création d'un nouvel homme plus ouvert sur l'universel (homo sovieticus). À l'ouest, le principe de la nationalité rejoignit le banc des accusés, au motif d'atrocités reliées aux deux guerres mondiales. Certains politiciens européens, comme De Gaulle, Schuman, de Gasperi ou Adenauer entrevirent la formation des États-Unis d'Europe comme le seul moyen de contrer la prolifération du nationalisme. Vers la fin des années dix-neuf cent quatre-vingt, l'équilibre atteint après la Seconde Guerre mondiale disparut subitement avec la chute du communisme et le démembrement de l'Union soviétique puis, dans son sillage, de celui de la Yougoslavie. La dissolution de ces puissances multinationales permit à plusieurs pays de reprendre leur autonomie nationale. L'État national réapparut alors comme une réponse à une tendance naturelle et comme un retour à ce qui l'avait 
constitué historiquement ${ }^{14}$. Les États baltes recouvrirent leur indépendance; l'Ukraine, la Biélorussie, la Géorgie, l'Arménie et les républiques islamiques se détachèrent de la Russie; la Slovénie et la Croatie se séparèrent de la Yougoslavie; la Tchécoslovaquie se scinda en deux pays distincts. Puis le processus s'élargit et fit resurgir, en Yougoslavie, la question de l'identité nationale des peuples de Bosnie, du Kosovo, de l'Albanie, de la Macédoine.

En 1939 déjà, Edvard Kardelj, bras droit slovène de Tito, avait écrit que la Yougoslavie n'aurait de sens pour les Slovènes que dans la mesure où leurs intérêts nationaux y seraient protégés. En 1991, les Slovènes eurent, pour la première fois depuis l'épisode lointain de la Carantanie, l'occasion de recréer un État autonome, beaucoup plus petit toutefois. C'est sans hésitation qu'ils y souscrivirent, en conformité avec leur aspiration séculaire.

Depuis la Seconde Guerre mondiale, en plus de leur existence nationale menacée par la Yougoslavie, les Slovènes furent soumis à un régime totalitaire d'idéologie athée. L'homme nouveau communiste rejetait tout Être supérieur et toute loi qui n'émanait pas de lui et qui n'était pas conforme à ses objectifs ou à ses intérêts. Dostoïevski aurait pu dire: sans Dieu, tout leur était permis. Dans ce système idéalisé, l'homme pouvait s'accommoder de tout ce qui correspondait à ses intérêts ou aux intérêts de la cause pour laquelle il combattait, puisqu'il se considérait lui-même comme le maître absolu de l'histoire. Cet homme, en refusant la transcendance, écartait la prétention que l'éternité fût la continuation mystérieuse de l'existence humaine. Pour lui, l'essentiel se résumait à ce monde-ci et à ce temps-ci, et ce n'est qu'à ce titre qu'il entrevoyait l'unique sens de sa vie et de ses activités. Marx avait déjà ajouté sa contribution à cette conscience immanente en affirmant que lui seul connaissait bien le sens et l'orientation de l'histoire, ainsi que la voie qui menait au grand soir, à l'accomplissement de

14. «La Gesellschaft selon Tönnies implique "un cercle d'hommes qui, comme dans la Gemeinschaft, vivent et habitent en paix les uns à côté des autres mais, au lieu d'être essentiellement unis, sont au contraire essentiellement séparés, et tandis que dans la Gemeinschaft ils restent unis malgré toutes les distinctions, ici ils restent distincts malgré tous les liens". Par conséquent, il ne s'y trouve pas d'activités qui puissent être déduites d'une unité existant a priori et nécessairement qui expriment la volonté et l'esprit de cette unité [...] Mais chacun est ici pour soi et dans un état d'hostilité visà-vis des autres. Les divers champs d'activité et de pouvoir sont fortement déterminés les uns par rapport aux autres, de sorte que chacun interdit aux autres tout contact et toute immixtion [...] Personne ne fera rien pour autrui à moins que ce ne soit en échange d'un service similaire ou d'une rétribution qu'il juge être l'équivalent de ce qu'il donne [...] Seule la perspective d'un profit peut l'amener à se défaire d'un bien qu'il possède » (Durkheim 1889, 3). 
cette histoire où se trouvait finalement le mystère du divin. Sous l'égide du socialisme marxiste, l'homme politique devenait un succédané du Dieu qui avait conduit, jusque là, la destinée des nations. Désormais, l'homme prendrait entre ses mains son avenir, qu'il façonnerait grâce à son activité politique révolutionnaire; c'est ainsi qu'il se rachèterait et qu'il se réaliserait grâce à la révolution ${ }^{15}$.

Dès que le régime communiste prit le pouvoir, il se comporta en fait comme l'héritier et le gérant du sacré et du bien suprême et se déclara réfractaire à la pratique religieuse. En Slovénie, différentes périodes se succédèrent durant les quarante-six ans d'existence d'un régime aux allures plus ou moins intolérantes envers l'expression religieuse tant individuelle que publique. L'apogée de la période d'intolérance à l'égard de l'Église ${ }^{16}$ et des croyants se situa dans les vingt premières années, de 1945 à 1965. Puis, succéda une courte accalmie d'apparence libérale, entre 1965 et 1972, suivie d'un intervalle d'instabilité idéologique et de déclin politique, caractérisé par

15. Les communistes ont présenté la révolution d'Octobre comme le modèle par excellence pour toute future révolution en Europe et dans le monde. La révolution française ayant constamment échappé au contrôle de ceux qui l'avaient déclenchée, jamais, par la suite, aucun homme ni aucun parti politique n'avaient su ou pu se la réapproprier. Lénine, au contraire, a façonné son propre parti autour de l'idée de révolution, et depuis novembre 1917, ce parti communiste se considère comme le seul héritier légitime de la révolution d'Octobre, car il est le seul à en comprendre le sens et la portée. Le parti s'arroge le droit de gouverner non parce qu'il détient ce mandat du peuple, mais parce qu'il a fait la révolution. Il veut ainsi démontrer empiriquement qu'il possède, par rapport aux autres partis, une connaissance supérieure de la légitimité historique et qu'il est le seul capable d'en assurer la réalisation. Sa perspicacité exceptionnelle l'élève au-dessus du commun et, par là-même, justifie son monopole politique. Le communisme, sous la férule de Lénine d'abord, puis de Staline, devient progressivement une des grandes illusions modernes. Il va conduire des nations vers une nouvelle période historique prétendument exemplaire. Si pour atteindre cet objectif, il a besoin de violence, il ne s'en prive pas, et du coup il tient la contre-révolution responsable de ses échecs puisque, en s'opposant à lui, elle ne comprend pas ou ne veut pas comprendre la nouvelle réalité (Rode 1997, 67).

16. Nous parlons ici tout particulièrement de l'Église catholique en raison du fait que, en 2007 encore, la grande majorité des Slovènes est catholique, soit $70 \%$ de la population totale. Le pourcentage des autres dénominations religieuses est minime: l'Église évangélique (1,1\%), l'Église orthodoxe (0,6\%) et l'Islam (0,6\%). Dans ce contexte, il est important de mentionner que l'Église, en tant qu'institution, n'a jamais fait sienne la tâche de confronter directement le pouvoir du parti communiste. Toutefois, elle fut pendant de longues décennies la seule force à conserver l'espace de liberté que la violence idéologique avait épargné. Malgré tout, le parti la voyait comme l'ennemi intérieur le plus menaçant de l'ordre socialiste et comme un danger constant. Aussi le pouvoir la tenait-elle en laisse économiquement, dans les domaines de la formation, de l'éducation et de l'accès aux média (Stres 2004b, 16). 
des tentatives réformistes favorisant un communisme "à visage humain » et un "eurocommunisme». Ces tendances, sans jamais prédominer vraiment, engendrèrent une certaine fragilité du régime et une relative tolérance.

La proximité de pays de l'Ouest influença le mode de vie de la Slovénie. Toutefois, l'idéologie marxiste demeura fondamentalement hostile à la foi, et l'athéisme actif imposé aux membres du Parti se perpétua jusqu'à la fin du régime. Pendant l'accalmie soi-disant libérale, le régime communiste yougoslave toléra plus ou moins la religion dans la mesure où elle demeurait une affaire individuelle et dans la mesure où son expression publique restait interdite. Quant au système scolaire, il fut durement marqué par le marxisme athée. En 1974 particulièrement, interdiction totale fut faite aux croyants de briguer un poste dans le domaine pédagogique; la police secrète surveillait étroitement les agissements de l'Église et de ses représentants, surtout les membres du clergé. L'article 174 de la constitution SFRJ ${ }^{17}$ stipulait que "la profession de la foi est libre et du ressort de l'individu ». En conséquence de quoi, la dimension publique, bien qu'essentielle à toute liberté, était exclue. Le même article 174 stipulait aussi que les communautés religieuses ${ }^{18}$ fussent séparées de l'État et il limitait leurs activités au domaine des activités et des cérémonies religieuses. Il était tout particulièrement défendu de tenir des écoles, sauf des petits séminaires. La constitution justifiait ses principes fondamentaux de la façon suivante: les prérogatives et les droits individuels sont limités non seulement par les prérogatives et les droits des autres, mais aussi par «les intérêts de la société socialiste ". Selon cette même constitution, "la formation et l'éducation sont fondées sur les acquis de la science contemporaine, et surtout sur le marxisme, source du socialisme scientifique ». De plus, le parti communiste, ou plutôt, l'union communiste, se voulait « l'idée directrice et la force politique» et «le détenteur et le promoteur principal de l'activité politique». Le régime actuel a mis un terme à la restriction sur la croyance limitée au domaine privé ${ }^{19}$, mais la manière de concevoir une telle relation

17. Socialistična federativna republika Jugoslavija.

18. Par communautés religieuses, nous entendons les différentes Églises ou religions et leurs constituants; nous ne l'entendons pas dans le sens restreint des congrégations ou des ordres religieux.

19. En 2008, dix-sept ans après la fin du régime communiste, la Slovénie compte quatre lycées privés (15-18 ans) et plusieurs garderies privées. Toutefois, chose étonnante, aucune école primaire-secondaire privée (7-15 ans) n'a encore vu le jour, même si on s’y prépare depuis plusieurs années. 
à l'égard de la foi et de l'Église, pendant presque cinquante ans, a créé des stéréotypes et des habitudes qui perdurent.

L'histoire européenne récente nous a servi certains exemples de territoires annexés par des régimes qui se sont constitués comme une fin en soi. Toute dictature s'érige en vérité absolue et exhorte le citoyen à ne plus se soucier du besoin de croire en quelqu'un d'autre ou en un au-delà. Elle ne donne pas non plus au citoyen - et même à tout son peuple — l'occasion ni même le droit d'exprimer publiquement sa foi personnelle, ni de garder un lien privilégié avec le sacré. C'est l'aspiration de l'être par le vide. Au fond, l'approche dictatoriale relativise toute foi, désormais perçue comme une rivale, sauf celle imposée par sa propre idéologie. L'être humain n'étant plus vu comme inviolable dans sa dignité transcendante, le pouvoir dictatorial le considère plutôt comme un moyen utile et nécessaire pour atteindre ses propres objectifs. Le communisme en Europe centrale, après 1945, s'était donné pour objectif d'extirper la foi religieuse du cœur humain et d'éradiquer tout symbole chrétien de la société, de la culture et de la tradition populaire, tout en caressant l'ambition avouée de créer un monde nouveau, une société sans classe, un homme qui serait finalement libre de se distancier socialement et religieusement de ses comportements passés. Un divorce radical se produisit effectivement entre l'État, les communautés religieuses de Yougoslavie et d'autres États européens socialistes. Scientifiquement dirigée, l'idéologie marxiste-léniniste, seule prétendument capable de connaître la vérité, refusait toute autre conception du monde, y compris la conception religieuse. Dès lors, la liberté de conscience avait surtout comme fonction de libérer la conscience de l'individu de ses superstitions religieuses. Le principe de la séparation de l'Église et de l'État, entendu au sens de combat idéologique politique, eut comme conséquence d'éliminer les communautés religieuses non seulement de l'État, mais aussi de la société civile et de les reléguer au secteur de la vie privée uniquement.

Cela s'est déroulé à trois niveaux : institutionnel, personnel et fonctionnel. Au niveau institutionnel, les organismes d'État et les institutions ne tolérèrent plus, dans leurs activités, les contenus, les formes et les signes religieux, et les détenteurs de fonctions dans les communautés religieuses furent, de facto, évincés de toute responsabilité institutionnelle étatique. Au niveau personnel, les croyants furent exclus des positions de leadership et même du domaine des professions, sous prétexte qu'ils n'étaient pas qualifiés pour exécuter certaines activités. Au niveau fonctionnel, le principe de la séparation entraîna la laïcisation de toutes les activités de l'État et de la société civile qui avaient une incidence directe sur l'individu : le social, la formation 
et l'éducation, la culture, les médias, l'économie, l'organisation politique, etc. (Šturm 2004, 221) ${ }^{20}$.

Si l'État, à titre de communauté politique dotée d'un pouvoir souverain, se considère lui-même comme le gardien et le garant des droits, il lui incombe en retour de veiller à ce que les citoyens se réalisent et qu'ils utilisent cette liberté pour leur épanouissement. Quand son approche anthropologique l'amène à privilégier le bien-être, le plaisir, la consommation, il laisse alors prédominer l'aspect matérialiste, utilitariste et hédoniste. Dans un tel cas la question de la liberté au service de la spiritualité retiendra peu son attention. Toutefois, tôt ou tard, la question du sacré et du transcendant refera surface, quelle que soit la culture de la nation, et lancera un appel impérieux pour le respect de la vie de l'individu et de la société, car le sacré s'inscrit - comme tout phénomène humain — dans le temps et dans l'espace et engendre inévitablement des répercussions.

Le respect des valeurs humaines souveraines ou des rituels demeurera toujours une preuve du respect qu'on accorde à toutes les autres valeurs humaines et qui sera leur meilleure garantie de survie. "Si un État totalitaire n'arrive pas à reconnaître la liberté d'expression religieuse et l'autonomie la plus intime de l'homme, il est fort probable qu'il ne respectera pas d'autres aspects ou d'autres espaces citoyens moins importants » (Stres 2001, 146). Tout système qui bafoue les droits humains voudra tôt ou tard s'immiscer dans la pensée, l'opinion, l'agir et les valeurs des gens. Inversement, toute résistance à la dictature commence d'abord dans le cœur profond de l'être, là où logent les valeurs. En retour, la liberté religieuse parfaite ne peut vraiment s'exprimer que dans un état réellement démocratique.

\section{Le sacré et la nationalité slovène}

Depuis douze siècles, la religion et l'identité nationale slovène ont tissé des liens très étroits. Toutefois, entre les deux, un point de division majeur s'est

20. Toutefois, dans les États européens modernes, un État laïque objectif et neutre par rapport à la religion et à la conception du monde ne privilégie aucune des deux, mais il ne les entrave pas non plus. Il n'enseigne ni ne refuse aucune philosophie individuelle, ni idéologie, ni morale. Il n'encourage ni n'interdit aucune doctrine: que ce soit le rationalisme, le matérialisme, le libéralisme, le marxisme, etc. La laïcité ne devient pas une idéologie officielle, ni une morale, ni une religion d'État. Les individus restent libres d'influence indue dans ce domaine. Dans des décisions qui ont un sens et un contenu religieux, l'État n'intervient que si cela s'avère nécessaire pour garantir la coexistence entre les individus et garantir le maintien des fondements de l'ordre social. Le devoir essentiel de l'État est d'assurer la neutralité et d'interdire l'immixtion dans la liberté des individus. 
glissé. En dépit de son apparence formelle libérale, le régime communiste façonnait un contenu a-libéral. La forme nationale slovène apparaissait libérale dans la séparation des pouvoirs tels que définis dans la constitution et dans les institutions, et libérale aussi dans une apparente conformité avec la libéralité historique, quoique souvent formelle, des civilisations européennes. L'attitude politico-philosophique est ainsi devenue un sacré nourri de collectivisme au niveau du contenu et d'individualisme au niveau de la forme. La tension nation-religion se manifeste toujours aujourd'hui depuis que la fin du socialisme sécularisé mis en place par le régime communiste, successeur du catholicisme, a exposé l'identité nationale au danger de l'auto-dégradation. Il est important, ici, de distinguer deux aspects de ce danger, lesquels sont à la fois d'origine moderne et d'origine historique chrétienne. C'est plutôt sous un angle chrétien que nous tenons à resituer la définition de la tradition et de la culture, d'une part, et le contenu d'origine qui assure le fondement de la civilisation occidentale, d'autre part ${ }^{21}$.

Le premier mai 2004, à l'instar de plusieurs pays européens, la Slovénie est devenue membre de l'Union Européenne, phénomène qui constitue, après sa sécession d'avec la Yougoslavie et son indépendance en 1991, un deuxième moment particulièrement marquant de son histoire récente. Malgré tout, même après son entrée dans l'Union, la nation slovène reste encore partiellement mal définie. Le point névralgique de toute crise d'identité, qu'elle soit nationale ou internationale, se situe dans la définition de l'ensemble des croyances qui regroupent une communauté particulière et lui fournissent les ressources nécessaires à l'existence commune, et à la survie des valeurs qui la font vivre. La reconnaissance de cet ensemble en lien

21. "Tout cela fait apparaître clairement que l'on ne peut pas penser édifier une authentique "maison commune" européenne en négligeant l'identité propre des peuples de notre continent. Il s'agit en effet d'une identité historique, culturelle et morale, avant même d'être géographique, économique ou politiqu; une identité constituée par un ensemble de valeurs universelles, que le christianisme a contribué à forger, acquérant ainsi un rôle non seulement historique, mais fondateur à l'égard de l'Europe. N'estil pas surprenant que l'Europe d'aujourd'hui, tandis qu'elle vise à se présenter comme une communauté de valeurs, semble toujours plus souvent contester le fait qu'il existe des valeurs universelles et absolues? Cette forme singulière d' "apostasie" d'elle-même, avant même que de Dieu, ne la pousse-t-elle pas à douter de sa propre identité ? De cette façon, on finit par répandre la conviction selon laquelle la "pondération des biens" est l'unique voie pour le discernement moral et que le bien commun est synonyme de compromis. En réalité, si le compromis peut constituer un équilibre légitime d'intérêts particuliers différents, il se transforme en mal commun chaque fois qu'il comporte des accords qui nuisent à la nature de l'homme » (Benoît XVI 2007, 3). 
avec le sacré est salutaire car il entraîne un ensemble d'applications sociales et politiques.

Le rapport entre la société moderne et l'État moderne est sans équivoque : les deux, apparemment de concert, ont écarté de la sphère publique la religion qui se réfère à l'au-delà et l'ont reléguée à l'immanence interpersonnelle. Il s'ensuit que la normativité de la religion n'est plus justifiée par l'affirmation ontologique et transcendante, mais par des ententes conventionnelles, donc humaines et sociales. Le concept de la religion chrétienne traditionnelle, exprimée en termes de croyances, de coutumes et de rituels, a pris fin officiellement en Slovénie lorsque le peuple s'est prononcé en faveur de la nouvelle constitution de la Nation démocratique en 1991. De relation verticale avec le sacré, la situation a basculé vers une relation horizontale qui s'est imposée comme le nouveau sacré, et elle s'est ouverte à la question suivante: quelle hypothétique religion supplantera dorénavant la religion classique ${ }^{22}$ ? La définition du concept de sécularisation proposée par Peter Berger et Thomas Luckmann (1967, 117-127), par exemple, est opérationnelle. La sécularisation constitue pour eux l'autonomisation progressive de secteurs sociaux qui échappent à la domination des significations et des institutions religieuses. Luckmann, religiologue d'ascendance slovène, est convaincu malgré tout que le christianisme en

22. Un dilemme au niveau social se pose en Slovénie entre la société et la religion civile comme l'explique Wasserstrom. Après la Seconde Guerre mondiale, la religion apparut comme sur son déclin, tant aux États-Unis qu'en Europe. De récents événements mondiaux avaient jeté un doute sur la pertinence de la croyance religieuse, et des tendances modernes avaient rendu déplacés les rituels religieux. C'est dans ce contexte que les carrières de Scholem, Eliade et Corbin — érudits du vingtième siècle dans leur domaine respectif du Judaïsme, de l'Histoire des religions et de l'Islam — convergèrent et révolutionnèrent la façon dont les gens réfléchissaient sur le fait religieux. Entre 1949 et 1978, les trois s'initièrent au cercle Eranos de Jung, à Ascona. C'est là que chacun à sa façon en est venu à identifier le symbolisme de l'expérience mystique comme élément central de sa tradition monothéiste. À ce sujet, Wasserstrom explore comment les trois en sont arrivés à renverser les approches traditionnelles de l'étude des religions, en restreignant l'accent mis sur la loi, le rituel et l'histoire sociale et en exaltant le rôle du mythe et du mysticisme. Selon lui, l'aspect le plus controversé de leur théorie de la religion réside dans le fait que leur approche minimise le caractère contraignant de la loi morale associée au monothéisme. Wasserstrom accorde une attention particulière à l'insistance fondamentale placée sur l'imagination symbolique et sur l'impact que cette orientation a eu sur leur jugement de l'histoire, de la politique et de l'éthique (voir Wasserstrom 1999). 
Slovénie demeure toujours la religion de base du territoire ${ }^{23}$, religion marquée par un christiano-théocentrisme difficile à expurger des travaux scientifiques. Malgré les efforts considérables déployés par des chercheurs ${ }^{24}$ pour forger le concept de modernité en tant que concept métaculturel et métahistorique, la notion de (post)modernité est encore et toujours rattachée à ses fondations ancestrales occidentales ${ }^{25}$.

Dans ce contexte, il convient de faire appel aux recherches internationales Aufbruch ${ }^{26}$, réalisées entre 1995 et 2000 dans les anciens États communistes. Leurs résultats nous montrent que la Slovénie n'appartient pas aux cultures croyantes de l'Europe de l'Est ou de l'Europe centrale, comme la Pologne, la Croatie, la Lituanie, l'Ukraine, la Transylvanie. Elle ne fait pas partie non plus des États athéisés comme l'ex-Allemagne de l'Est et la Tchéquie, mais elle fait plutôt partie des États polarisés où deux groupes équivalents de croyants et de non-croyants s'affirment l'un contre l'autre. De plus, même dans le groupe des croyants on peut déceler une sous-polarisation entre le groupe des personnellement croyants et fidèles à l'enseignement de l'Église et le groupe où apparaissent différents degrés, plus ou moins souples, de foi et d'appartenance. Selon ce sondage Aufbruch, à la question "Croyez-vous en Dieu?", $53 \%$ des interrogés ont répondu affirmativement et $12 \%$ négativement; $33 \%$ ne croyaient pas en Dieu mais à l'existence d'un principe suprême. La plupart d'entre eux accordent encore une

23. Pourtant des réflexions comme celles d'Alain Touraine et de Jean Baubérot poussent le débat dans le sens d'un dépassement des conceptions qui situent l'approche classique religion/tradition versus sécularisation/modernité dans la discontinuité ou la rupture, pour mieux souligner les effets de continuité; la modernité ne s'en trouve pas moins préservée et sa réalité continue d'être postulée, voire démontrée à la lumière de la diversité des circonstances dans lesquelles on observe ses effets, entre les contextes nationaux de France, des États-Unis, etc. Les travaux de Luckmann (1967 et 1990) expliquent bien cette transition, tout en soulignant certaines des caractéristiques symboliques du Nouvel ge concernant la signification ultime de l'existence des institutions publiques vers la "sphère du privé ".

24. Entre autres, Janez Juhant, Janez Markež, Bojan Žalec.

25. En même temps, selon Luckmann (1990), le mouvement du Nouvel ge, présent aussi en Slovénie, serait plutôt un résultat du déplacement des systèmes comme le syncrétisme et la présence d'une idéologie un peu vague. Les institutions primaires, comme l'État et l'Église, n'ont plus beaucoup de crédibilité aux yeux des populations occidentales et elles sont souvent coupées de la réalité des individus. La spiritualité humaine et la recherche d'un sens à la vie s'expriment désormais dans de nombreuses institutions secondaires.

26. Aufbruch est une agence internationale de sondage, d'analyse et de communication. La recherche en Slovénie a été coordonnée par Paul M. Zulehner, professeur de théologie pastorale à l'Université de Vienne. 
grande importance au mariage religieux, au baptême et aux funérailles religieuses; $20 \%$ de l'ensemble de la population assiste régulièrement à la messe dominicale et $30 \%$ aux grandes fêtes; parmi les croyants, $32 \%$ prient tous les jours, $25 \%$ une fois par semaine, $12 \%$ une fois par mois, $9 \%$ plus rarement. S'agissant du dogme, les Slovènes croyants sont plutôt libéraux; seulement $9 \%$ acceptent la doctrine de l'Église dans son intégralité (Pucelj 2003, 52-53). Selon les dernières recherches, effectuées entre 2002 et 2006, le nombre de croyants est en croissance modeste.

Toutefois, une autre question se pose : comment situer l'identité nationale slovène par rapport à la chrétienté, autrefois intimement liée à l'édification de la nation? Une des caractéristiques de cette dernière est d'avoir été fondée dans l'espace idéologique du socialisme de $\mathrm{Krek}^{27}$, dont le point de vue social-chrétien s'écartait du marxisme. Cette forme de socialisme fut, dès ses débuts, véhiculée par la religion. Plus tard, la sécularisation est arrivée par le biais du communisme, et, en même temps, est apparue une modernité travestie qui a supprimé la transcendance de la religion dorénavant transformée en immanence structurée. Le marxisme reflétait une immanence religieuse messianique qui, en bout de ligne, a vidé de son sens le lieu de cohérence sociale. L'individualisation de la religion en Slovénie ne s'est pas déroulée selon un processus propre à la modernité, mais plutôt comme un processus pré-moderne agrémenté d'un changement d'hégémonies idéologiques. Autrement dit, le fondement religieux comme catégorie de civilisation s'est estompé progressivement.

En Slovénie, nous pouvons parler d'un processus qui, au cours de la dernière moitié du vingtième siècle, s'est déroulé en trois temps, si bien que, après les chambardements révolutionnaires apportés par les deux guerres mondiales, dont l'une était de caractère impérialiste, et l'autre de caractère national-socialiste, le fondement social-religieux collectiviste en est arrivé à son point de rupture. Identifiée à la deuxième phase, cette rupture, tout

27. Parce qu'il considérait le paysan comme le pilier du peuple slovène, Janez Evanglist Krek (1865-1917) créa un système d'entraide sociale avec caisse d'épargne et caisse de prêt. En 1901, il publia sa plus importante œuvre scientifique: Socializem. Ce livre de 600 pages fut le premier de son genre en langue slovène. Il fut un penseur majeur sur la question de la nation slovène. Comme il ne voulait pas s'aligner sur les chrétiens-sociaux allemands, chez qui il décelait des tendances nationalistes et chauvinistes, Krek s'orienta, du temps de la monarchie austro-hongroise, vers la Croatie et la Serbie, pour la création d'un espace unifié des Slaves du Sud, reconnu plus tard comme la première Yougoslavie. Sur ses dernières années, il abandonna le point de vue du légitimisme, tendance royaliste française qui expliquait l'origine divine du pouvoir, pour choisir le point de vue démocratique de la souveraineté nationale. 
de suite après la Seconde Guerre mondiale, n'a pas été suivie d'une individualisation moderne mais d'une nouvelle collectivisation qui a duré un demi-siècle et qui s'est dotée, avec le temps, de tout un ensemble d'attributs profanes et athées (voir Luckmann 1967 et 1990). Si bien que la sécularisation qui s'en est suivie n'a pas procuré au citoyen l'individualisation, mais un succédané sacral de nature collective. De société religieuse d'origine cléricale, la société s'est métamorphosée en une société athée où le phénomène religieux a vécu une aliénation induite par les nouveaux clercs. On aurait pu y voir une ressemblance avec la fonction d'émancipation propre à l'esprit du capitalisme si cette société n'avait pas scié la branche de la religiosité d'origine qui lui tenait lieu de support. Alors que la doctrine capitaliste a essayé de tirer profit des fondements religieux, quoique dans une forme sécularisée dont il conservait certaines valeurs, l'esprit du nouveau collectivisme, soutenu quant à lui par le communisme, a voulu les écarter. Cette deuxième phase a pris fin à la suite d'un événement externe que l'on identifie à la chute du mur de Berlin. Lors de la troisième phase, l'État slovène indépendant s'est revêtu de formes libérales, mais, en même temps, la tendance pré-moderne et non-libérale de la période de Krek s'est fortement implantée. Puis, les répressions religieuses formelles liées au régime communiste cessèrent progressivement et, partant, l'individualisation religieuse prit le relais.

\section{Conclusion}

Que la nation slovène actuelle - et son instrument qu'est l'État - soit une formation artificielle est un fait incontestable. Une nation est une communauté qui, en cohérence avec le contrat social, a besoin d'un système de sens, d'un système de croyances ou d'une religion civile, d'un outil propre à rassembler les groupes d'intérêt partageant un même objectif national. La nation a besoin de son propre mythe qui, à sa façon, transcende l'immanence nationale. La nation slovène postmoderne a refusé un droit de dénominateur commun à la foi classique et à la religion traditionnelle. De plus, le prestige d'antan, qui semblait nimber la Narodno Osvobodilna Borba (Lutte pour la libération nationale) depuis le début de la révolution communiste, n'est plus guère à l'honneur aujourd'hui : au moins la moitié de la nation refuse ce mythe. Donc, ne demeurent que la culture et la langue qui sont aptes, mais insuffisantes, pour façonner une nation. Ainsi, l'État slovène et, avec lui, la nation slovène, se sont soumis aux effets du postmodernisme en raison de leur propre incapacité à vivre au niveau de leur 
identité. En se livrant au polycentrisme des valeurs et du plus petit dénominateur commun de convivialité sur leur territoire, les citoyens se reconnaissent aujourd'hui davantage dans la mondialisation que dans les idéaux anciens de leur propre nation (Markež 2001, 288). Nous nous rapprochons ici davantage des propos de Tönnies qui dit que la vie des grandes agglomérations sociales "serait caractérisée par un développement progressif de l'individualisme, dont l'action de l'État ne pourrait prévenir que pour un temps et par des procédés artificiels les effets dispersifs » (Durkheim 1889, 8). Serait-ce là une des raisons qui ont amené la Slovénie à quitter la Yougoslavie? Mais alors pourquoi vouloir se retrouver dans la communauté encore plus grande qu'est l'Union Européenne? Serait-ce alors rejoindre le point de vue de Durkheim pour qui «la vie des grandes agglomérations sociales est tout aussi naturelle que celles des petits agrégats bien qu'elle soit autre » (Durkheim 1889, 8) ?

Il semble que le champ de la nouvelle systématisation globale des valeurs de la nation slovène et le polycentrisme postmoderne des valeurs soient devenus une nouvelle porte d'entrée pour la chrétienté post-traditionnelle et pour la régénération de son contenu. Mais, s'il est possible d'affirmer que le problème national slovène se situe au niveau des valeurs et "du sacré tel qu'il est maintenant » après la fin du collectivisme sécularisé, on peut se poser la question suivante : quel rôle pourraient jouer, dans ce processus, les systèmes religieux traditionnels que sont le catholicisme et le protestantisme, c'est-à-dire, la chrétienté institutionnalisée ? Cette question s'adresse aussi à l'islam, depuis qu'un nombre relativement important de musulmans sont venus d'autres républiques de l'ex-Yougoslavie, de Bosnie particulièrement. Les influences du polycentrisme sur les institutions religieuses vont probablement ressembler beaucoup aux influences guidées par le désir de garder l'idéologie d'une nation moderne. Même si en Slovénie la tradition catholique demeure encore très présente, elle perd rapidement de sa pertinence sociale, alors même que les défis de la mondialisation, de l'information globale et de la consommation court-circuitent l'argumentation avec laquelle elle défendait autrefois les valeurs nationales. S'agissant de la religion, la situation de la nation slovène ressemble généralement à celle d'autres nations européennes. Sauf que plusieurs d'entre elles ont déjà consolidé leur identité, se rendant ainsi moins vulnérables. La nation slovène, au contraire, en raison d'une plus grande fragilité liée à son âge relativement jeune, a encore plus besoin que d'autres nations de protection vis-à-vis de l'individualisme, de la fragmentation, du relativisme, de l'apathie, 
de l'indifférence et du scepticisme postmodernes. Pour réaliser son processus de formation et de subsistance, une nation ne peut, pour sa stabilité, faire l'économie de la recherche et de la découverte de ses fondements sacrés, la relation avec le sacré étant, par définition, un guide vers le sens, vers l'espoir et vers la question du critère objectif pour ce qui est juste ou pas, de ce qui est bien ou mal. En cela, même si on accuse les institutions religieuses slovènes d'être passéistes, leur point de vue, quant au contenu existentiel, s'avère encore pertinent : ce sont elles qui ont fondé la civilisation européenne et qui ont grandement aidé les Slovènes à se développer sur leur territoire actuel.

\section{Références}

Armstrong, J. (1997), "Religious Nationalism and Collective Violence», Nations and Nationalism, 3/4, p. 597-606.

BENoîT xvi (2007), "L'Europe a-t-elle perdu son âme?", Osservatore romano, 2975 , p. 3.

Berger, P. et T. LuCKMANN (1967), «Aspects sociologiques du pluralisme », Archives de sociologie des religions, 23, p. 117-127.

Durkheim, É. (1889), “Communauté et société selon Tönnies », édition électronique réalisée à partir d'un texte extrait de la Revue de philosophie, 27, p. 416-422.

(1907), «Cours sur les origines de la vie religieuse », édition électronique réalisée à partir d'un texte extrait de la Revue de philosophie, $7 / 5$, p. 528-539.

Hollis, M. (1995), Soziales Handeln, Berlin, Akademie-Verlag.

HonNenfelder, L. (1996), «Person und Menschenwürde », dans L. HonNENfelder et G. Kreiger, Philosophische Propädeutik, Bd. 2. Ethik, Paderborn, F. Schöningh, p. 234-248.

Hribar, T. (1986), "Razlika med božjim in svetim ", Nova revija, 48-49, p. $934-948$.

JeAn-PAul II (1980), «La visite à l'UNESCO», La Documentation catholique, 1788, p. 603-609.

(1995), "Un accord international doit traiter des droits des nations », La Documentation catholique, 2125, p. 917-923.

JHUANT, J. (2006), Človek v iskanju svoje podobe, Ljubljana, Claritas.

Jhuant, J. et B. Žalec, (2006), Person and God, Berlin, LIT Verlag. 
Kos, J. (1996), Duhovna Zgodovina Slovencev, Ljubljana, Slovenska matica. Luckmann, T. (1967), The Invisible Religion: The Problem of Religion in Modern Society, New York, Macmillan.

(1990), "Shrinking Transcendence, Expanding Religion", Sociological Analysis, 50/2, p. 127-138.

MARKeš, J. (2001), "Religija in nacionalna identiteta ", dans P. KVATERNIK, V Prelomnih časih, Ljubljana, Kvaternik, p. 277-290.

New Encyclopedia Britannica, The (1985), « The Study and Classification of Religions ", vol. 26.

Отто, R. (1993) [allemand 1917], «Sveto. O iracionalnem v ideji Božjega in njegovem razmerju do racionalnega ", Ljubljana, Nova revija, p. 13-62.

PucelJ, J. (2003), "Slovenski katoličani in Evropska unija ", Tretji dan (krščanska revija za dubovnost in kulturo), 52-53, p. 49-56.

Rode, F. (1997), Za čast dežele, Ljubljana, Družina.

Rousseau, J.-J. (1915) [1772], "Considérations sur le gouvernement de la Pologne ", dans C. E. Vaughan, The Political Writings of Jean-Jacques Rousseau, II, Cambridge, Cambridge University Press, p. 369-434.

SloterdiJK, P. (1998), Sphären I. Blasen, Frankfurt, Suhrkamp Verlag.

Smith, A. D. (2000), "The Sacred Dimensions of Nationalism », Millennium: Journal of International Politics, 29/3, p. 791-814. (2005) [anglais 2001], Nacionalizem - Teorija, idelologija, zgodovina / trad. par B. Cajnko, Ljubljana, Krt.

Stres, A. (1991), Oseba in družba, Celje, Mohorjeva družba.

(2001), "Moralna moč in nemoč ideje človekovega dostojanstva », Bogoslovni vestnik, 2, p. 129-152.

(2004), «Svoboda veroizpovedi kot temeljna človekova pravica v slovenski tranziciji », Bogoslovni vestnik, 1, p. 15-22.

ŠTURM, L. (2004), Sveto in svetno - pravni vidiki verske svobode, Celje, Mohorjeva družba.

TAmarut, A. (2005), Kršćanski pogled na čovjeka, Zagreb, Glas koncila.

Tiluich, P. (1951), Systematic Theology, vol. 1. Reason and Revelation, Being and God, Chicago, The University of Chicago Press.

Vergote, A. (19952, 1983), Religion, foi, incroyance, Bruxelles, Mardaga. 
VODOPIVEC, P. (2007), « Kulturno-duhovne razmere na slovenskem v 19. stoletju », Bogoslovni vestnik, 1, p. 9-18.

Wasserstrom, S. M. (1999), Religion after Religion, New Jersey, Princeton University Press.

\section{Résumé}

La notion du sacré s'étend de la réalité substantielle à l'illusion fonctionnelle. Selon nous, la personne humaine en tant qu'être transcendant est un être sacré pour qui la religiosité est un phénomène irréductible; cette personne est aussi un être social. La culture concrétise cette double réalité dans un État, dans une nation. Après 1945, en plus de voir leur existence nationale menacée par une Yougoslavie impérialiste, les Slovènes subirent un régime communiste totalitaire qui voulait créer une société sans classes et un homme libre, mais cette société est plutôt devenue un «sacré» nourri de collectivisme et d'individualisme. L'État et la nation slovènes, en refusant un droit de dénominateur commun à la religion traditionnelle et au mythe de la Narodno Osvobodilna Borna (Lutte pour la libération nationale), se sont soumis aux effets du postmodernisme en raison de leur propre incapacité à vivre leur identité. En se livrant au polycentrisme des valeurs et au plus petit dénominateur commun de la convivialité, les citoyens se reconnaissent aujourd'hui davantage dans la mondialisation que dans les idéaux de leur propre nation.

\section{Abstract}

The notion of the "sacred" can apply to substantial reality as well as the most functional of illusions. The paper starts from the premise that humans are transcendental and, as such, sacred beings for whom religiosity is irrefutable; they are also social beings. Cultures translate this double reality into states and nations. After 1945, Slovenes did not only see their national existence threatened by an imperialist Yugoslavia; they also bore the brunt of a totalitarian communist regime bent on creating a classless society and a free man. However, this soon turned into a "sacred" enterprise to push collectivism and individualism. By denying traditional religion and Narodno Osvobodilna Borna (national liberation struggle) their role as common identity denominators, the Slovene state and the nation fell under the sway of postmodernism because of their failure to express their real identity. By surrendering to the polycentrism of values and to the least common denominator of congeniality, Slovene citizens now recognize themselves more in the processes of globalization than in the aspirations of their own nation. 